\title{
Motivación del personal sanitario en la formación continuada sobre tratamiento quimioterápico
}

\author{
J osé Ramón García Fernández y Francisco López Berenguel. \\ Hospital General Básico de Baza. Unidad de Hematología. Servicio Andaluz de Salud.
}

\begin{abstract}
Introducción : El tratamiento quimioterápico es una parte fundamental en la actividad de un centro hospitalario. Sin embargo en los hospitales comarcales el desarrollo de esta actividad es especialmente complejo. La formación continuada postgraduada juega un papel central en la optimización del proceso, y la motivación para participar en ella es asimismo fundamental.
\end{abstract}

Material y métodos : Describimos el proceso de realización de un curso de formación continuada en un hospital general básico, así como la motivación del personal para que participe activamente en la formación continuada, con varias técnicas: información exhaustiva previa, implicación directa como docente, beneficios curriculares y económicos, etc. Previo al curso se plantearon 4 objetivos: $1^{\circ}$ asist encia de todo el personal de enfermería directamente implicado, $2^{\circ}$ aumento de conocimientos generales, $3^{\circ}$ adquisición de habilidades especiales y $4^{\circ}$ centrar el curso en la asistencia integral al enfermo oncológico. Los objetivos se evaluaron mediante: 1.-lista de asistencia,

2 y 3-cuestionario anónimo previo y al finalizar el curso y 4.-valoración subjetiva.

Resultados: Asistieron al curso de forma regu lar un total de 53 perso nas so bre una plantilla "diana" de 456, fundamentalmente diplomados universitarios de en fermería (DUE). Los objetivos propuestos se cumplieron respectivamente en un: $75 \%, 50 \%, 75 \%$ y $100 \%$.

CONCL USIONES: El personal sanitario más motivado en la formación continuada en oncología básica es: diplomados en enfermería, médicos internos residentes y curiosamente técnicos especialistas de laboratorio. El contacto habitual con la preparación y administración

Correspondencia:

J osé Ramón García Fernández

Banco de Sangre (Laboratorio)

Hospital General Básico de Baza.

Carretera de Murcia s/n

Baza (Granada) 18800

Correo electrónico:jrdallas@wanadoo.es de tratamientos citostáticos fue la principal mo tivación para asistir al curso. Las diversas técnicas de motivación empleadas so lo fuero $n$ eficaces en el pers onal p red isp uest o "a prio ri” a la realización del curso.

Palabras clave:

Formación continuada, quimioterapia

Motivation of health staff in training about chemotherapy treatment.

Introduction: Pharmacological treatment with cytostatic agents is an important part of hospital practice. In community hospitals this activity is specially difficult to develop, by the tendency of the staff to consider this specific of the third level hospitals. Postgraduate continuing education plays a central role in the process, and the motivation to participate in these is also essential.

Design and methods: We describe the development of an activity of postgraduate continuing education in a community hospital. The interest of the experience is how motivate health staff in order to participate actively in work out organizated learning with several methods: previous exhaustive information, possibility of participate as teacher, educative and economic advantages,.. . First of all we settled 4 objetives: $1^{\circ}$ : att endance of all health staff related, $2^{\circ}$ : real increase of knowledge, $3^{\circ}$ : acquirement of specific habilities and $4^{\circ}$ : that the course must be based on the int egral aid of oncologic patient. The objetives were evaluated by: $1^{\circ}$ : list of attendance, $2^{\circ}$ and $3^{\circ}$ : anonymous questionnaire realized before and after the course, and $4^{\circ}$ : subjetive appraisal.

Results: 53 hospital's health staff assisted continually to the course, above all nurses, of a "target" population of 456. The objetives propposed were reached respectively in a : $75 \%, 50 \%, 75 \%$ and $100 \%$.

Conclusion: The health staff more motivated in postgraduate continuing education in basic oncology 
are: postgraduate physicians, nurses and curiously laboratory technicians. Habitual contact with chemotherapy treatments was the most important factor to assist to the course. The methods used in order to motivate the attendance only were successful by the staff motivated "a priori" in the performance the course.

Key words:

Pstgraduated continuing education. Chemotherapy.

\section{INTRODUCCIÓN}

El tratamiento quimioterápico es una parte fundamental en la actividad de un centro hospitalario, sea cual sea su nivel asistencial. Aunque la complejidad de los tratamientos asumidos varía en función del nivel de asistencial del mismo. En los grandes centros hospitalarios la administración de fármacos citostáticos es una función bien establecida y asumida como tal por el personal sanitario, sin embargo en los hospitales comarcales el desarrollo de esta actividad es más complejo ya que existe una cierta tendencia, no solo en los pacientes sino también en el personal del hospital, a considerar el tratamiento oncológico como propio y exclusivo de los grandes hospitales regionales. Para vencer dichas resistencias y que tanto las indicaciones como el procedimiento sean adecuadas, es imprescindible la colaboración de todo el personal hospitalario implicado en alguna parte del procedimiento, para asegurar una continuidad de la "cadena". A mayores conocimientos la posibilidad de error disminuye, siendo esta inversamente proporional a los conocimientos adquiridos por el personal que participa directamente en su indicación, preparación, transporte y administración. La existencia de un sistema de información periódica forma parte de los procedimientos estándares mínimos en los centros en los que se administran citostáticos ${ }^{(1,2)}$. La tan nombrada pero muchas veces infravalorada "formación médica continuada" juega un papel central en la optimización del proceso. Para ello es indispensable que el médico que trabaja en ellas interactúe con sus colegas, aprenda conjuntamente e introduzca efectivas iniciativas ${ }^{(3)}$ y es esencial que a la vez que programamos cualquier actividad de formación seamos capaces de motivar a los profesionales sanitarios a los que va dirigida para que participen activamente en la misma.

El objeto del presente trabajo es valorar los resul- tados obtenidos en un curso de formación continuada sobre tratamiento quimioterápico, en el que se persiguen unos objetivos concretos aparte del aumento "bruto" de conocimientos. El contenido del curso se programó en función de las necesidades observadas en el curso anterior realizado en 1997 en el que se observó una discordancia entre los conocimientos impartidos y los requeridos por el personal; así como una relativa falta de interés de los diplomados en enfermería respecto a los conocimientos impartidos por facultativos especialistas, mientras que la atención era total cuando el docente pertenecía a su misma categoría. Por ello es importante evaluar el resultado de un curso para impartir posteriores ediciones del mismo, ya que la formación continuada debe ser "continuada" por definición. En estos casos debemos tener en cuenta la máxima: "cuando algo es un fracaso absoluto, siempre nos puede servir como mal ejemplo". Se planteó así un curso diseñado desde el principio para ser más atrayente para los discentes.

\section{MA TER IAL Y METODOS}

El Hospital General Básico de Baza (Granada) es un centro público del Servicio Andaluz de Salud, (S.A.S.). Dispone de 169 camas hospitalarias, con una pdolación asignada de 132.314 personas en el año $2000^{(4)}$. E n él se administran de forma regular ditostáticos desde diciembre de $1996{ }^{(5)}$, ya sea en régimen dehospital de día camo de ingreso hospitalario.

Al inicio de su actividad se realizó un curso de formación básica impartido principalmente por médicos especialistas. E $n$ dicho curso aunque se plantearon unos objetivos muy modestos, el único que se consiguió fue iniciar la actividad de la unidad. Al evaluar "a posteriori" el curso se objetivó que ni los docentes ni los contenidos eran los adecuados a los objetivos planteados.

Dentro del programa de formación continuada de la unidad de hematología y con el fin de potenciar la actividad de la misma y mejorar la formación del personal se programó en el año 2000 un curso oficial autorizado y financiado por el Servicio Andaluz de Salud sobre: "Actualización en tratamientos citostáticos" de 30 horas de duración, dividido en 20 temas (tabla 1) dirigido a personal sanitario, segunda edición del curso anteriormente citado. Al esbozar y dar forma a dicho curso se dirigió específicamente a una población "diana", formada por los diplomados y auxiliares de enfermería del hospital; aunque no se limitó el acceso al mismo a ninguna otra categoría 
TABLA 1: TEMARIO DEL CURSO “ACTUALIZACIÓN EN TRATAMIENTOS CITOSTÁTICOS”

Tema1: Introducción ¿qué es un citostático?. Concepto de enferme dad neoplásica.

Tema 2: Clasificación de fármacos citostáticos.

Tema 3: Fármacos adyuvantes y otros fármacos utilizados en las enfermedades neoplásicas.

Tema 4: El hospital de día onco-hematológico: fundamentos y utilización.

Tema 5: Vías periféricas y centrales: indicaciones, utilización y complicaciones.

Tema 6: Principales protocolos de uso de poliquimioterapia en oncohematología.

Tema 7: El enfermo oncológico (I).

Tema 8: El enfermo oncológico (II).

Tema 9: Protocolo de extravasaciones y derrames.

Tema 10: Protocolo de tratamiento del síndrome febril en el paciente neutropénico.
Tema 11: Urgencias oncológicas.

Tema 12: Terapia transfusional: generalidades.

Tema 13: El procedimiento transfusional en el Hospital General.

Básico de Baza: protocolos y funcionamiento del banco de sangre.

Tema 14: Reacciones transfusionales inmediatas y tardías.

Protocolo de reacción transfusional.

Tema 15: Tratamiento de residuos: generalidades y tratamiento específico de residuos quimioterápicos.

Tema 16: Hospitalización a domicilio (I).

Tema 17: Hospitalización a domicilio (II).

Tema 18: Trasplante de médula ósea (I): fundamentos teóricos y procedimientos.

Tema 19: Trasplante de médula ósea (II): indicaciones y resultados. Tema 20: Trasplante de médula ósea (III): protocolos de enfermería. profesional. Los objetivos fundamentales que se plantearon fueron los siguientes:

1.- Asistencia al curso del personal de enfermería directamente implicado en la administración de citostáticos (adscritos a la unidad de hematología y planta de hospitalización de cirugía) y secundariamente del personal de otras unidades de hospitalización y de urgencias.

2.- Aumento de conocimientos generales sobre el área impartida.

3.- Adquisición de conocimientos y habilidades sobre técnicas específicas (uso de reservorios venosos de acceso subcutáneo, administración de citostáticos y manejo de extravasaciones).

4.- Orientar el curso globalmente hacia el tratamiento holístico del enfermo oncológico. Al considerar el tratamiento quimi oterápico debemos considerar fundamentalmente al enfermo y sus circunstancias. El estudio de los diversos fármacos, los protocolos de tratamiento, el procesamiento de excretas y muestra biológicas sol o tiene sentido si lo centramos en la curación/mejoría/ayuda a un paciente concre to. Eso es un acercamiento holístico, se trató siempre de hablar "del enfermo", lo que es mejor para él y su calidad de vida.

Para intentar conseguir los objetivos se progr amó un curso impartido por personal del hospital y de otr os centros hospital ari ios. Se procuró (y se consiguió) que todo el personal docente tuvier a experiencia personal y directa en el área impartida, para conseguir una información realista y no académica. La titulación fue la siguiente auxiliares de enfermería: 1; diplomados universitarios en enfermería: 7 y facultativos especialistas de ár ea: 3; uno de ellos afecto de una neoplasia en tratamiento con citostáticos, que impartió el curso como paciente a la vez que como médico. Se pretendía una titulación de los docentes lo más similar a la población diana del curso.

No se impuso limitación de plazas ni restricción según categorías profesionales. Los asistentes contestaron un cuestionario abierto de 30 preguntas antes del inicio del curso y al finalizarlo, sobre los aspectos más importantes del mismo. Se analizó el porcentaje de inscritos según categorías y servicios, así como las respuestas y comentarios de los cuestionarios, de forma global y específicamente según los objetivos planteados.

La motivación del personal a inscribirse primero y asistir después al curso se realizó de la siguiente forma:

1.-Se anunció personal mente la programación del curso a todo el personal directamente implicado, no solo a través de carteles y envíos postales personalizados

2.-Se ofreció la posibilidad de colaborar como docentes a todo el personal relacionado. Se ofrecía a la vez ayuda para el desarrollo de los temas: material docente, soporte informático, dotación económica y acreditación docente. De tal forma que aunque no aceptara ser docente, sí se despertara interés por el curso de tal forma que sí asistiera como discente.

3.-Anunciar explícitamente la asistencia de personal con experiencia directa en la materia a impartir.

4.-Insisti r periódicamente al personal relacionado sobre la existencia del curso y el interés que puede tener el mismo para su desarrollo profesional.

\section{RESULTADOS:}

Se inscribieron y asistieron al curso de forma regular un total de 53 personas sobre una plantilla "diana" de 456 (todo el personal sanitario del hospi- 
tal). La titulación de los asistentes fue la siguiente: auxiliares de enfermería: 4; técnicos especialistas de Iaboratorio: 8; ATS/DUE: 31; Médicos internos residentes: 6; facultativos especialistas de área: 4. Asistieron el $100 \%$ de los diplomados en enfermería adscritos a la unidad de hematología y a la planta de cirugía. La asistencia de facultativos especialistas y auxiliares de clínica fue testimonial $(<5 \%$ de personal de cada categoría). Resultaron Ilamativos 2 hechos: el primero fue que no asistió personal sanitario extrahospitalario del área de salud y el segundo que aproximadamente un $50 \%$ de la plantilla de técnicos especialistas de laboratorio acudió al curso. Se pudieron valorar en paralelo un total de 46 cuestionarios de 30 preguntas con una media de respuestas acertadas al inicio del curso de 19,98 (desviación estándar: 3,25) y al final de 26,00 (desviación estándar: 2,16 ). Para verificar si se había producido un incremento real de conocimientos se realizó un test de Student para muestras independientes (ya que al ser los cuestionarios anónimos no se pudieron obtener datos apareados) con varianzas distintas, ( $p<0,001$ para 82,36 grados de libertad), con lo que se evidenció un aumento real de los mismos estadísticamente significativo.

Los temas que suscitaron un mayor interés fueron: extravasaciones y derrames, tratamiento de residuos y muestras biológicas, enfoque psicológico del paciente oncológico, visión personal de la enfermedad neoplásica y el uso de reservorios venosos de acceso subcutáneo. Medidos en términos de : mayor número de cuestiones planteadas durante el desarrollo del curso, con un $100 \%$ de respuesta acertadas en el cuestionario final

En nuestro caso de los 4 objetivos planteados al inicio del curso, la valoración de su cumplimiento es la siguiente:

Objetivo 1: (75\%) Asistencia regular de un $100 \%$ del personal de enfermería directamente implicado, con asistencia mínima de enfermeros/as del resto de servicios hospitalarios. No se inscribió personal extrahospitalario.

Objetivo 2: (50 \%) Aumento real, en valores absolutos: [26-19,98/30=6,02/30 $(p<0,001)]$ de conocimientos sobre el área impartida

Objetivo 3: (75\%) Adquisición real de conocimientos espeáficos: uso de reservorios venosos de acceso subautáneo, administración de citostáticos y man jio de extravasaciones.

Objetivo 4: (100\%) Valoración de la importancia de un acercamiento holístico al enfermo oncológico. Esta es una apreciación subjetiva y por tanto no objetivable. Se basa en el hecho de que en todo momento el protagonista del curso, el centro de atención fue el enfermo. Todo el programa desarroIlado basado en técnicas y fármacos se focalizó en el paciente al que se aplicaban. Induso, como ya se ha referido, intervino como ponente un médico que expuso su experiencia personal sobre el tema desde el punto de vista del personal sanitario por un lado y del enfermo por otro.

\section{DISCUSIÓN:}

En el ámbito de la formación continuada se pretende que el beneficiario último de la misma sea el usuario del sistema, en este caso el paciente, ya que la mejora de conocimientos y habilidades del personal debe traducirse en una mejoría de la calidad asistencial. Sin embargo esta afirmación tiene sus matices, ya que en los cursos impartidos por univer sidades, fundaciones, sindicatos, sociedades científi cas y otras instituciones, el beneficiario es el discente, que asiste al curso por diversas motivaciones: curricular, desarrollo profesional y personal, interés científico, etc. En el caso de los cursos impartidos en los centros hospitalarios, a este interés del discente se une el interés del docente en impartir una formación al personal con el que desarrolla su actividad clínica diaria, para que esta sea lo más eficiente posible. Por esto en la formación continuada realizada en los centros hospitalarios, al realizar programas de formación, es esencial implicar de forma adecuada al personal al que va dirigida la acción, para que el desarrollo del programa sea el correcto. Es necesario motivar al personal: primero para que se inscriban al curso, segundo para que aquellas personas más capacitadas y dispuestas impartan parte del curso como docentes y tercero, para que una vez iniciado el curso asistan al mayor número posible de ponencias y participen activamente en ellas.

Las conclusiones obtenidas de la valoración de esta experiencia son las siguientes:

A) El personal sanitario más motivado en la formación continuada en oncología básica pertenece a las siguientes categorías profesionales: diplomados en enfermería, médicos internos residentes y curiosamente técnicos especialistas de laboratorio. Esto último se explica por la adscripción en los hospitales comarcales, de los facultativos especialistas en hematología-hemoterapia a los servicios de análisis dínicos, donde desarrollan sus funciones junto a los técnicos especialistas de laboratorio, trasladándose 
esto en un interés de los mismos hacia el área puramente dínica.

B) EI contacto habitual con la preparación y administración de tratamientos citostáticos fue la principal motivación para asistir al curso. Con ausencia total de personal de atención primaria y una asistencia del $100 \%$ del personal de enfermería adscrito a la unidad de hematología y de la planta de hospitalización de cirugía; que es el que habitualmente realiza estos tratamientos.

C) Las diversas técnicas de motivación empleadas solo fueron eficaces en el personal predispuesto "a priori" a la realización del curso, no sirvieron en el personal sanitario no directamente implicado en el tema.

Sin embargo la principal lección extraída de la realización de este curso en el seno de una formación continuada que tenemos que desarrollar día a día, es la importancia de implicar a todo el personal del hospital en cualquier actuación que se vaya a realizar en el mismo. Esta teoría general es totalmente válida en el caso del tratamiento quimioterápico. Si realmente queremos administrar citostáticos de forma efectiva en un centro (no me refiero a eficiente) y por consiguiente tratar adecuadamente a pacientes oncológicos, debemos contar con todo el personal del mismo.

Según la experiencia desarrollada en este proceso, los principales aspectos a considerar para motivar al personal sanitario a participar activamente en los procesos de formación continuada son los siguientes:

1.-Considerar siempre la opinión de todo el personal con el que se trabaja: todos estamos trabajando en el mismo sitio y nuestros intereses son comunes, luego toda opinión debe ser tomada en cuenta y valorada.

2.-Tener en cuenta especialmente la opinión del personal de enfermería, que es el que soporta el grueso de la actividad asistencial.

3.- En la preparación del curso, el ofrecer a todo el personal directa o indirectamente implicado y con experiencia directa en el tema (a todos) la posibilidad de intervenir como docente, para que se sientan parte directa de la actividad; a la vez que se les ofre ce la ayuda necesaria para realizar la exposición.

4.-Intentar que la titulación de los docentes sea, en general, lo más similar posible a la de los discentes; ya que un auxiliar de clínica escuchará siempre con más atención a otro de su misma categoría profesional que a un facultativo especialista de un hospital universitario.

5.-Los docentes siempre tienen que disertar sobre asuntos sobre los que tienen experiencia directa en su actividad profesional diaria.

Los cursos de formación continuada ofrecen de esta forma una oportunidad única al poder implicar al personal sanitario como docente a la vez que como destinatario del curso.

\section{BIBLIOGRAFÍA}

1.- Mateu J, Massó-Muniesa J , Clopés A, Òdena $E$, Trullàs $M$. Consideraciones en el manejo de la extravasación de citostáticos. Farm Hosp. 1997; 21: 187-194.

2.- Prat A (coordinador). Recomanacions per a la manipulació de medicaments antineoplàsticas als centres hospitalaris. Barcelona: Servei Català de la Salut. Generalitat de Catalunya, 1994; 1-55.

3.- Pardell H. Formación médica continuada, ¿individual o institucional?. Med Clin (Barc) 2001; 116: 698-700.

4.- Memoria anual 2000. Hospital General Básico de Baza (Granada). Servicio Andaluz de Salud.

5.- García Fernández J R, López Berenguel F, Moreno PoloF, Pérez Salvador A, HaroPujada C, Marin Rødríguez A. E valuación del tratamiento quimioterápico realizado en la Unidad de Hematología del H.G.B. de Baza en un periodo de 3 años. Actas de las XX J ornadas de la AAHH; 2000, abril 28-29; Ú beda. A sociación Andaluza de Hematología y $\mathrm{H}$ emoterapia. 